International Mathematical Forum, 2, 2007, no. 39, 1921 - 1925

\title{
Essential Ideals and Finsler Modules
}

\author{
A. Taghavi and M. Jafarzadeh \\ Department of Mathematics \\ University of Mazandaran, Babolsar, Iran \\ taghavi@nit.ac.ir
}

\begin{abstract}
In this paper, the notion of associated ( essential) ideal submodule in Finsler module over $C^{*}$-algebras is introduced. Moreover, it is shown that if essential ideal submodule $V_{I}$ is a Hilbert $I$-module, then $V$ is itself a Hilbert $A$-module.
\end{abstract}

Mathematics Subject Classification: Primary 46C50, 46L08

Keywords: $\mathrm{C}^{*}$-algebra, essential ideal, Finsler module, Hilbert $C^{*}$-module

\section{Introduction}

A (right) Hilbert $C^{*}$-module over a $C^{*}$-algebra $A$ is a right $A$-module $V$ equipped with $A$-valued inner product $\langle.,$.$\rangle which is a A$-linear in the second and conjugate linear in the first variable such that $V$ is a Banach space with the norm $\|x\|=\|\langle x, x\rangle\|^{\frac{1}{2}}$ (see [3]).

Finsler modules over $C^{*}$-algebras are generalization of Hilbert $C^{*}$-modules that first investigated in [5]. Let $A$ be a $C^{*}$-algebra and $A^{+}$denote the set of positive elements of a $\mathrm{C}^{*}$-algebra $A$. Let $V$ be a right module over $C^{*}$-algebra $A$ and the map $\rho: V \rightarrow A^{+}$satisfies the following condition.

(i) the map $\|\cdot\|_{V}: x \rightarrow\|\rho(x)\|$ is a Banach space norm on $\mathrm{V}$; and

(ii) $\rho(x a)^{2}=a^{*} \rho(x)^{2} a$ for all $a \in A$ and $x \in V$.

Then $V$ is called a Finsler module over $C^{*}$-algebra $A$.

A Finsler module over $C^{*}$-algebra $A$ is said to be full if the linear span $\left\{\rho(x)^{2} ; x \in V\right\}$ denoted by $\mathcal{F}(V)$ is dense in $A$.

Ideal submodules in Hilbert $\mathrm{C}^{*}$-modules are investigated in [2] and [6]. In [5] Phillips and Weaver proved that if $(V, \rho)$ is a Finsler module over $C^{*}$-algebra $A$ such that $\rho$ satisfies the parallelogram law

$$
\rho(v+w)^{2}+\rho(v-w)^{2}=2 \rho(v)^{2}+2 \rho(w)^{2},
$$


for $v, w \in V$, then $V$ is a Hilbert $A$-module. In this paper, the notion of associated (essential) ideal submodule in Finsler modules over $C^{*}$-algebras is introduced and it is shown that if $\rho$ satisfies the parallelogram law only on a essential ideal submodule $V_{I}$ then $V$ is a Hilbert $A$-module, that is, if essential ideal submodule $V_{I}$ is a Hilbert $I$-module, then $V$ is itself a Hilbert $A$-module.

\section{Preliminaries}

Definition 2.1. let $V$ be a Finsler modules over $C^{\star}$-algebra $A$, and let $I$ be an ideal in $A$. The associated ideal submodule $V_{I}$ is defined by

$$
V_{I}=[V I]^{-}=[\{v b: v \in V, b \in I\}]^{-}
$$

(the closed linear span of the action of $I$ on $V$ ).

Clearly, $V_{I}$ is a closed submodule of $V$. It can be also regarded as a Finsler module over $I$.

In general, there exist closed submodules which are not ideal submodule. For instance, if a $C^{*}$-algebra $A$ is regarded as a Hilbert $A$-module (with the inner product $\langle a, b\rangle=a^{*} b$ ), then ideal submodules of $A$ are precisely ideals in $A$, while closed submodules of $A$ are closed right ideals in $A$.

We arise some properties of ideal submodules. Following results are already known of $([2],[1])$. let $V$ be a Finsler module over $\mathrm{C}^{*}$-algebra $A$, and $I$ be an ideal of $A$. By application of Hewitt-Cohen factorization theorem ([4], Theorem 4.1,[6], proposition 2.31) it is easy to that $V_{I}=V I=\{v b: v \in V, b \in$ $I\}$. If $V$ be a full Finsler module over $A, V_{I}$ will be full over $I$ [1, Lemma 2.2].

Remark 2.2. let $V$ be a Finsler module and $I$ be an ideal of $A$, and $V_{I}$ be associated ideal submodule. Define by $q: V \rightarrow \frac{V}{V_{I}}$ and $\pi: A \rightarrow \frac{A}{I}$ the quotient maps. By definition right action of $\frac{A}{I}$ on linear space $\frac{V}{V_{I}}$ with $q(v) \pi(a)=q(v a), \frac{V}{V_{I}}$ will be a $\frac{A}{I}$-module and by [5 Lemma 12], $\frac{V}{V_{I}}$ is a Finsler $\frac{A}{I}$ module with norm Finsler $\rho_{\frac{A}{I}}(q(v))=\pi\left(\rho_{A}(v)\right)$. Then $\rho_{\frac{A}{I}}(q(V))=\pi\left(\rho_{A}(V)\right)$, so $\left[\rho_{\frac{A}{I}}(q(V)]=\pi\left(\left[\rho_{A}(V)\right]\right)\right.$.

In addition, $\frac{V}{V_{I}}$ is a full Finsler $\frac{A}{I}$-module if and only if $V$ is full. This follows at once from the evident equality $\left[\rho_{\frac{A}{I}}(q(V)]=\pi\left(\left[\rho_{A}(V)\right]\right)\right.$.

With similar argument of [2 p. 4], if $X$ be a closed submodule of $V, J$ be an ideal of $A$ such that $\rho(V) \subseteq J$, then $\frac{V}{X}$ with module action $q(x) \pi(a)=q(x a)$ is a $\frac{A}{J}$-module iff $X=V_{J}$. Note that smallest of such ideals is $A$-linear hull $\left(\rho(V)^{2}\right)$. 


\section{3 main results}

Definition 3.1. Let $I$ be an ideal of $C^{*}$-algebra $A$, define $I^{\perp}=\{a \in A: a I=$ $0\}$ (that is ideal of $A$ ). I is essential if $I^{\perp}=\{0\}$, that is equivalent $I \cap J \neq\{0\}$ for all closed ideal $J$ of $A$.

The following lemma is a much similar relative of Lemma 1.10 of [2]. (Enough that replacing $I$ with $I^{+}$denoted positive elements of $I$ ).

Lemma 3.2. Let $I$ be an ideal in a $C^{*}$-algebra $A$. The following condition are mutually equivalent:

(a) $I$ is an essential ideal in $A$

(b) $\|a\|=\sup _{b \in I^{+},\|b\| \leq 1}(\|a b\|), \forall a \in A$

(c) $\|a\|=\sup _{b \in I^{+},\|b\| \leq 1}(\|b a\|), \forall a \in A$

(d) $\|a\|=\sup _{b \in I^{+},\|b\| \leq 1}(\|b a b\|), \forall a \in A^{+}$.

Theorem 3.3. let $V$ be a Finsler module and $I$ be an essential ideal of $A$, and $V_{I}$ be (associated) ideal submodule. Then $\|v\|=\sup _{b \in I^{+},\|b\| \leq 1}(\|v b\|), \forall v \in$ $V$. Conversely, if $V$ is a full Finsler module over $C^{*}$-algebra $A$ and $\|v\|=$ $\sup _{b \in I^{+},\|b\| \leq 1}(\|v b\|), \forall v \in V$ respect to some ideal $I$ of $A$, then $I$ is an essential ideal in $A$.

Proof . Let $I$ be an essential ideal in $A$, then $\forall v \in V$

$$
\begin{aligned}
\|v\|^{2} & =\|\rho(v)\|^{2}=\sup _{b \in I^{+},\|b\| \leq 1}\left(\left\|b \rho(v)^{2} b\right\|\right) \\
& =\sup _{b \in I^{+},\|b\| \leq 1}\left(\left\|\rho(v b)^{2}\right\|\right) \\
& =\sup _{b \in I^{+},\|b\| \leq 1}(\|v b\|)^{2},
\end{aligned}
$$

by definition of norm Finsler and Lemma 3.2.

To prove the converse, suppose that $V$ is a full Finsler module and $I$ is not essential so that $I^{\perp} \neq\{0\}$. Take any $c \in I^{\perp}, c \neq 0$. By $[1$ proof Theorem 3.2 (iii)], there exists $v \in V$ such that $v c \neq 0$. Now by hypothesis of theorem we have

$$
\begin{aligned}
\|v c\| & =\sup _{b \in I^{+},\|b\| \leq 1}(\|(v c) b\|) \\
& =\sup _{b \in I^{+},\|b\| \leq 1}(\|v(c b)\|)=0 .
\end{aligned}
$$

Means $v c=0$, that is a contradiction. So $I$ is an essential ideal of $A$.

Recall that a Finsler $A$-module $V$ with map $\rho$ is a Hilbert module if $\rho$ produce a $A$-valued inner product $<.$, . $>$ on $V$ such that $V$ is a Hilbert $A$-module and $\rho(x)=<x, x>^{1 / 2}$ for all $x \in V$.

Theorem 3.4. Let $V$ be a Finsler module of commutative $C^{*}$-algebra $A$ and $I$ be an essential ideal of $A$. If essential ideal submodule $V_{I}$ is a Hilbert 
I-module, then $V$ is itself a Hilbert A-module.

Proof . Let $\rho$ be norm Finsler map over $V$. Note that essential ideal submodule $V_{I}$ is a Finsler $I$-module with map $\left.\rho\right|_{V_{I}}$. Hence $\rho$ satisfies in parallelogram law on $V_{I}$. Therefore, for all $v, w \in V$ and $a \in I$, we have

$$
\begin{aligned}
& \rho \quad(v a+w a)^{2}+\rho(v a-w a)^{2}-2 \rho(v a)^{2}-2 \rho(w a)^{2}=0 \\
& \left.\Leftrightarrow \quad a^{*}\left(\rho(v+w)^{2}+\rho(v-w)^{2}\right)-2 \rho(v)^{2}-2 \rho(w)^{2}\right) a=0 \\
& \Leftrightarrow \quad\left(\rho(v+w)^{2}+\rho(v-w)^{2}-2 \rho(v)^{2}-2 \rho(w)^{2}\right) a^{*} a=0 .
\end{aligned}
$$

Now Lemma 3.2(b), show that $\left\|\rho(v+w)^{2}+\rho(v-w)^{2}-2 \rho(v)^{2}-2 \rho(w)^{2}\right\|=0$. Hence, $\rho(v+w)^{2}+\rho(v-w)^{2}=2 \rho(v)^{2}+2 \rho(w)^{2}$ means norm Finsler $\rho$ satisfies in parallelogram law on $V$, so by [5 lemma 12] $V$ is a Hilbert $A$-module.

Recall that if $A, B$, and $D$ are $C^{*}$-algebra, and if homomorphisms $\phi: A \rightarrow$ $D$ and $\psi: B \rightarrow D$ are given, then the $C^{*}$-algebra $A \oplus_{D} B$ is defined as

$$
A \oplus_{D} B=\{(a, b) \in A \oplus B: \phi(a)=\psi(b)\} .
$$

We use the same notation for modules, Banach spaces, etc.

Let $A$ be a $C^{*}$-algebra. By [5 lemmas 10 and 11] $A$ has a unique maximal commutative ideal $I_{0}$ and a closed ideal $J$ such that $I_{0} \bigcap J=\{0\}$ and $\frac{A}{J}$ is

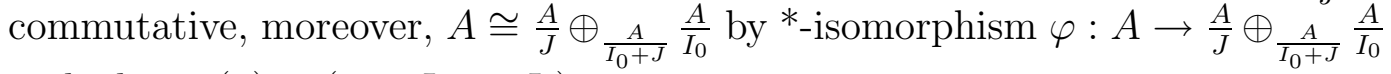
such that $\varphi(a)=\left(a+J, a+I_{0}\right)$.

Lemma 3.5. Let $A$ be a $C^{*}$-algebra, $I_{0}, J$ and $\varphi$ be as in above argument, $I$ be an essential ideal in $C^{*}$-algebra $A$ if and only if $\frac{I}{J}$ and $\frac{I}{I_{0}}$ are essential ideal in $C^{*}$-algebras $\frac{A}{J}$ and $\frac{A}{I_{0}}$ respectively.

Proof . It is clear that quotient map $\pi: A \rightarrow \frac{A}{J}$ is a ${ }^{*}$-surjective homomorphism. It is enough to show that $\pi(K) \bigcap \frac{I}{J} \neq\{0\}$ for arbitrary closed ideal $K$ of $A$ such that $K \bigcap J \neq K$. Suppose that $\pi(K)$ is a non zero closed ideal of $\frac{A}{J}$. There exists $k \in K-J$, so we have

$$
0 \neq \varphi(k)=\left(k+J, k+I_{0}\right) \in \pi(K) \oplus_{\frac{A}{I_{0}+J}} \frac{A}{I_{0}} .
$$

So $\pi(K) \oplus_{\frac{A}{I_{0}+J}} \frac{A}{I_{0}}$ is a non zero closed ideal of $\frac{A}{J} \oplus_{\frac{A}{I_{0}+J}} \frac{A}{I_{0}}$. Since $I \cong \frac{I}{J} \oplus_{\frac{A}{I_{0}+J}} \frac{I}{I_{0}}$ is essential ideal in $C^{*}$-algebra $A$. Now we have $\{0\} \neq \pi(K) \bigcap \frac{I}{J}$. Hence $\frac{I}{J}$ is a essential ideal of $\frac{A}{J}$. Similar statement is true for $\frac{I}{I_{0}}$.

Conversely, let $a \in I^{\perp}$. Then $a c=o$ for every $c \in I$. Thus $a c \in J$ and $a c \in I_{0}$. It implies that $a+J \in\left(\frac{I}{J}\right)^{\perp}$ and $a+I_{0} \in\left(\frac{I}{I_{0}}\right)^{\perp}$. Therefore $a \in I_{0} \bigcap J=\{0\}$ because $\frac{I}{J}$ and $\frac{I}{I_{0}}$ are essential ideal. Hence in view of the 
mention of above this Lemma $a=0$. Consequently, $I^{\perp}=\{0\}$, that means $I$ is an essential ideal.

Theorem 3.6. Let $V$ be a Finsler module over $C^{*}$-algebra $A$ and $I$ be an essential ideal of $A$. If essential ideal submodule $V_{I}$ is a Hilbert $I$-module, then $V$ is itself a Hilbert $A$-module.

Proof . By Theorem 17 of [5], we can write $V \cong V_{1} \oplus_{V_{0}} V_{2}$, where $V_{2}$ and $V_{0}$ are Hilbert $\frac{A}{I_{0}}$ and $\frac{A}{I_{0}+J}$ modules resp., and $V_{1}$ is a Finsler module over commutative $\mathrm{C}^{*}$-algebra $\frac{A}{J}$. Also, we note that $\rho_{V}\left(x_{1}, x_{2}\right)=\left(\rho_{V_{1}}\left(x_{1}\right), \rho_{V_{2}}\left(x_{2}\right)\right)$, for every $x_{1} \in V_{1}$ and $x_{2} \in V_{2}[5$, Lemma 16]. Now by Lemma 3.5 and Theorem $3.4, V_{1}$ is a Hilbert module over $C^{*}$-algebra $\frac{A}{J}$. Consequently, $V$ is a Hilbert module over $C^{*}$-algebra $A$.

Remark 3.7. Essentiality can not be dropped. For instance, let $V$ be a non- Hilbert Finsler module and $I=0$ so is not essential. $V_{I}=0$ is a Hilbert $I$-module but $V$ is not.

\section{References}

1. M. Amyari and A. Niknam, On homomorphisms of Finsler modules, Intern. Math. Journal, vol. 3, No. 3 (2003), 277-281.

2. D. Bakic and B. Guljas, On a class of module maps of Hilbert $C^{*}$ modules, Mathematica communications, 7(2002), no.2 177-192.

3. E. C. Lance, Hilbert $C^{*}$-modules, LMS Lecture Note Series 210, Cambridge University Press (1995).

4. G. K. Pedersen, Factorization in $C^{*}$-algebras, Exposition Math., 16(1998), No 2, 145-156.

5. N.C. Phillips, N. Weaver, Modules with Norms which take values in a $\mathrm{C}^{*}$-algebra, Pacific journal of mathematics, vol. 185, no. 1, (1998).

6. I. Raeburn and D. P. Williams, Morita equivalence and continuous-trace $C^{*}$-algebras, Mathematical surveys and Monographs AMS 60 (1998).

Received: December 5, 2006 